\title{
The impact of non-urologic drugs on sexual function in men
}

\author{
Ferdinando Fusco, Marco Franco, Nicola Longo, Alessandro Palmieri, Vincenzo Mirone \\ Department of Neurosciences, Reproductive Science and Odontostomatology Federico II University, Naples, Italy.
}

\begin{abstract}
Summary Sexual dysfunctions have commonly been reported as the resulting side effects of many drugs. To understand the impact of a single drug, the mechanism of action of the most commonly prescribed drugs and the physiological mechanisms of sexual function have to be taken into dual consideration. Psychotropic drugs (Antidepressants, Antipsychotics and Antiepileptic) in particular result in both short and long-term effects on sexual function. Antihypertensive drugs have also produced evidence certifying their role in determining sexual dysfunction. Patients affected with sexual dysfunction are often aged and assume several drugs and, while Iatrogenic sexual dysfunction is prevalent in men, urological drugs are not the only drugs to be held accountable. Many different drugs acting on different sites and with several mechanisms of action can induce sexual dysfunction.

The drug classes involved are widely diffused and frequently assumed in combination therapies.
\end{abstract}

KEY wORDS: Sexual dysfunctions; Psychotropic drugs; Antihypertensive drugs.

Submitted 17 February 2014; Accepted 28 February 2014

\section{INTRODUCTION}

Sexual function is our physiological capacity to experience desire, arousal and orgasm. Male sexual activity is a multifaceted activity, involving complex interactions between the nervous system, the endocrine system, the vascular system and a variety of structures that are instrumental in sexual excitement, intercourse and satisfaction (1). As defined by Kaplan, this process consists of three phases: desire, arousal (excitement) and orgasm (2).

However, the division between the three is arbitrary and only assists in organizing clinical and research oriented problems involving sexuality. In clinical practice sexual desire, arousal and orgasmic difficulties more often than not coexist, suggesting an integration of phases.

In males, arousal basically the ability to achieve and maintain an erection. The orgasm is the mental and physical phenomenon that signifies the climax of coital activity and is accompanied by the ejaculation of seminal fluid (3). Normal sexual functioning requires not only an ability to interact with others (i.e. a certain level of social skills) but also a combination of different physiological factors, such as the integrity of the genitalia, the reliable co-ordination of blood flow, the activation of various smooth and skeletal muscles and the stimulation of local secretions (3)

Sexual dysfunction can result from a wide variety of psychological and physical causes. Pathophysiology of sexual dysfunction involves derangements in the levels of sex hormones and neurotransmitters (4), and different drugs affect sexual function in different ways depending on their mechanism of action. Drugs that affect libido usually act on the central nervous system and may reduce desire by causing sedation or hormonal disturbance. Likewise, drugs that interfere with the autonomic system will have negative effects on erectile function, ejaculation and orgasm (5). Many drug classes, other than "prostatic" drugs, have the potential to interfere with the physiology of sexual function. Knowledge of the normal biology of sexual function may allow one to predict whether a medication might potentially cause sexual dysfunctions. Furthermore, clinical experience acquired on different drug classes and molecules can confirm this potential. The purpose of this article is to review the impact of non-urologic drugs on sexual function in men.

\section{MATERIALS AND METHOdS}

In June 2013 a literature search was conducted from Medline to identify original articles published up to this date with no time limitation and analysis was conducted on previously published reviews and any other relevant articles suitable for the purposes of this review. The search was limited to articles published in English and was conducted by combining the following terms: ED and/or sexual dysfunction; aexual dysfunction and/or drugs, and or antidepressant, antiepileptics, antihypertensive, antipsycothic, antiparkinsonian, proton pump inhibitors, recreational drugs. The retrieval was then extended to the reference list and to the related articles. In the case of the availability of more than one publica-

No conflict of interest declared 
tion sharing the same information, the most recently published article was included in this review. Two independent reviewers evaluated the output listings to identify the references matching the selection criteria and checked each article to ensure consideration of further relevant published articles from the respective reference lists.

\section{Drugs that affect sexual function}

Several drug classes are reported to interfere with sexual function. Evidence was found for medications that differed greatly both for their indications and mechanisms of action, e.g.: antihypertensive, as beta blockers and thiazides, acting on peripheral blood flow and the sympathetic autonomous nervous system; proton pump inhibitors, acting on testosterone metabolism; drugs acting on the central nervous system as antidepressants, antiepileptics and antipsychotics, with their central mechanism of action on ions channels or nervous transmission (Table 1).

\section{Antidepressants}

Treatment-emergent sexual dysfunction caused by antidepressants is a considerable issue with a large variation across compounds (6). This is primarily due to the activation of 5-HT2 receptors which inhibit both noradrenergic and dopaminergic transmission. Generally, antidepressants with fewer 5-HT2 effects tend to cause less treatment- emergent sexual dysfunctions (7). Depression itself can cause sexual problems, thus it is sometimes difficult to recognize what is causing any occurring sexual dysfunction (8). It is therefore useful to try to get some idea of a person's sexual functioning prior to starting antidepressants. Serretti et al. (6) showed in a recent work on depressed men treated with antidepressants, that there was a significantly higher rate of total and specific treatment-emergent SD and specific phases of dysfunction compared with placebo for the following drugs in decreasing order of impact: sertraline, venlafaxine, citalopram, paroxetine, fluoxetine, imipramine, phenelzine, duloxetine, escitalopram, and fluvoxamine, with SD ranging in $25.8 \%$ to $80.3 \%$ of patients. No significant difference with placebo was found for the following antidepressants: agomelatine, amineptine, bupropion, moclobemide, mirtazapine, and nefazodone.

In conclusion the use of antidepressant therapy associated with the depression itself, has to be carefully considered in patients with a history of sexual dysfunction. As different antidepressants may have a different impact on sexual function, in the case of antidepressantinduced SD, a switch to an alternative antidepressant may be worth trying.

\section{Anti-epileptics}

Epileptic men may experience hormonal changes and altered levels of biologically active testosterone; changes that could alter semen quality and sexual function. In addition, $22 \%$ to $67 \%$ of men with epilepsy have been found to have reduced sexual interest and an impaired quality of sex life (9) Moreover, men with epilepsy are at a five-fold higher risk of erectile dysfunction (ED) than men without epilepsy (10).

Alterations in male sexual and reproductive parameters may also be due to treatment with antiepileptic drugs (AEDs) to control seizures $(11,12)$.

Carbamazepine: Adverse effects of carbamazepine (CBZ) use including impaired hepatic P450 enzyme system function and changes in serum sex hormone have been reported. Moreover, epileptic men taking CBZ present altered semen quality, ED, and a reduction in coital frequency (9). These AEDs related effects are explicable if considered as sexual hormonal changes.

Levetiracetam: The relationship between the older antiepileptic drugs (AEDs) and sexual dysfunction has long been known and is likely to be related to sexual hormonal changes. Instead, rare reports on sexual disorders related to new AEDs suggest the possibility of complex and poorly understood mechanisms as well as alterations of the central nervous system neurotransmitters

Table 1.

Different classes and single drugs causing sexual dysfunction.

\begin{tabular}{|l|l|}
\hline Medication class & Subclasses and single drugs \\
\hline Antidepressants & $\begin{array}{l}\text { Tricyclic antidepressants } \\
\text { Selective serotonin reuptake inhibitors (SSRIs) } \\
\text { Monoamine oxidase inhibitors (MAOIs) } \\
\text { Viloxazine, nefazodone, venlafaxine, reboxetine, mirtazepine, trazodone, duloxetine }\end{array}$ \\
\hline Antipsychotics & $\begin{array}{l}\text { Typical: aliphatic phenothiazines (e.g. chlorpromazine), thioridazine } \\
\text { Atypical: risperidone, quetiapine, aripiprazole olanzapine clozapine }\end{array}$ \\
\hline Antihypertensives & $\begin{array}{l}\text { Thiazide diuretics } \\
\text { Beta blockers }\end{array}$ \\
\hline Anti-parkinsonian drugs & $\begin{array}{l}\text { L-dopa } \\
\text { SSRIs }\end{array}$ \\
\hline Recreational drugs & $\begin{array}{l}\text { Psychostimulants, amphetamine, ecstasy, crystal methamphetamine, alcohol, anabolic steroids, } \\
\text { cannabis opiates, poppers, tobacco }\end{array}$ \\
\hline Proton pump inhibitors & Esomeprazole \\
\hline
\end{tabular}


such as glutamate, serotonin, and dopamine. In this regard Calabrò et al. (13) reported two men affected by epilepsy in which levetiracetam (LEV) intake appeared to induce a severe loss of libido and anhedonia.

Topiramate: Common side effects of Topiramate (TPM) comprise of fatigue, somnolence, dizziness, paresthesias and loss of appetite, but sexual dysfunctions including ED and anorgasmia are rarely reported $(14,15)$ Civardi et al. hypothesized that the inhibition of carbonic anhydrase could interfere with production of VIP and nitric oxide, two known intracavernosal vasoactive compounds that play a key role in the peripheral erectile mechanism, leading to a reduction in genital blood flow (16).

Indeed, Calabrò et al. (17) have suggested that TPMinduced ED may be secondary to a blockage of AMPA receptors with inhibition of the glutamatergic pathway, whereas glutamate is considered a candidate neurotransmitter of reflexive erection. To support this hypothesis, recent studies have shown the presence of both AMPA and NMDA glutamatergic receptor subunits in the lumbosacral spinal cord. Moreover, AMPA and NMDA receptor antagonists are known to block reflexive erection (18). In conclusion, the data reported in literature confirms the alteration of hormonal levels as mechanism of sexual dysfunction in men taking antiepileptic drugs and shows possible different mechanisms for the sexual effects caused by new anti-epileptics drugs.

\section{Antihypertensives}

Several reports collectively spanning more than three decades indicate that $2.4 \%-58 \%$ of hypertensive males experience one or more symptoms of sexual dysfunction of varying degrees of severity during antihypertensive drug therapy. It is also true, however, that hypertensive patients experience sexual dysfunction prior to taking medication, when compared to normotensive subjects. This finding, while quite consistent with the physiologic changes noted in hypertensive individuals, is often neglected in the overall assessment of subjects and in the formulation of a therapeutic scheme (19). The different anti hypertensive drugs have peculiar mechanisms through which sexual dysfunction is caused.

Beta blocker: Beta blockers (e.g., atenolol and propranolol) may potentially impact sexual functioning through a variety of mechanisms, including a reduction in central sympathetic outflow, impairment of vasodilatation of the corpora cavernosa, effects on luteinizing hormone and testosterone secretion, and a tendency to produce sedation or depression thereby causing a loss of libido (19).

An Italian study (20) on sexual activity and plasma testosterone in hypertensive men, evaluated the effects of the beta 1-selective beta-blocker atenolol on sexual activity and plasma testosterone levels in newly diagnosed, previously untreated essential hypertensive, sexually healthy men. One of the proposed mechanisms inducing sexual dysfunction is represented by an inhibition of the sympathetic nervous system which is involved in the integration of erection, emission and ejaculation in the regulation of luteinising-hormone secretion and the stimulation of release of testosterone (21). In this study atenolol significantly reduced plasma testosterone values thus confirming previous observations about the depression in testosterone levels in patients receiving this beta-blocker $(22,23)$. In contrast, a recent important review by Ko et al. (24) showed that the conventional wisdom that beta-blocker therapy is associated with substantial risks of sexual dysfunction is not supported by data from clinical trials and that the risk of sexual dysfunction is only minimally increased. The risk of these adverse effects, therefore, should be seen within the context of the documented benefits of these medications. Moreover, Nebivolol seems to have a beneficial effect on ED, possibly due to increased nitric oxide availability (25). The possible occurrence of ED with Metoprolol has, for the most part, been attributed to the prejudice (the so called "Hawthorne effect") on the "easy incidence" of this side-effect in hypertensive men. Since the etiology of this ED is largely psychological, it is not surprising that placebo is as effective as a PDE5inhibitor, namely tadalafil, in reversing this side effect (26). Thiazides: The associations that have been noted between the clinical use of the thiazide diuretics and impairment of male sexual performance remain enigmatic. A Medical Research Council Working Party in 1981 reported a 16\% incidence of impotence in patients taking thiazide diuretics after 12 weeks of treatment (Medical Research Council Working Party, 1981). A later study of diuretics in mild hypertension showed a significant increase in sexual dysfunction compared with placebo. The disorders noted included a reduction in libido, difficulty in obtaining and maintaining erection and problems with ejaculation. Patients on diuretics were 2 to 6 times more likely to experience sexual dysfunction than those on placebo (27). Recent studies investigated aspects of the male sexual dysfunction in hypertensive men following treatment with thiazide diuretics. The results suggest that penile erectile defects and decrements in sexual activities are specifically related to thiazide administration. A rodent model of thiazide-induced male sexual dysfunction, that documents dose-related impairment by hydrochlorothiazide of penile erectile reflexes and male copulatory performance is available (28). The etiology of thiazide-induced male sexual dysfunction is hypothesized to result from alterations in sodium excretion that alter afferent renal nerve input to hypothalamic areas regulating male sexual responses.

\section{Other antihypertensive drugs}

Calcium channel blockers (CCB) and angiotensin-converting enzyme (ACE) inhibitors have not been associated with major negative impact on ED. It has also been suggested that angiotensin II type 1 receptor blockers (ARB) would affect sexual function less than other antihypertensive treatment. Some data even suggests that sexual function and ED may improve during treatment with ARB (29).

In conclusion, two groups of antihypertensive drugs were examined in this review which have produced evidence on their role in determining sexual dysfunction. However, in both groups of drugs, individual mechanisms of action through which such alterations of sexual function was perpetrated, have not yet been well defined.

\section{Antipsychotics}

Human sexual function is affected in many different ways by schizophrenia and the antipsychotic drugs used in its 
treatment. The evaluation of the effects of antipsychotics on sexual function in patients with schizophrenia is also complex because the deleterious effects of conventional antipsychotics are superimposed on the effects of the disease itself (30). Studies have suggested that, although antipsychotic drugs often restore sexual desire lost due to schizophrenia, they may impair patients' sexual performance $(30,31)$. In a recent study evaluating a sample of male psychotic patients, both sexual dysfunction and hyperprolactinemia were very prevalent (32). Based on their findings in 72 patients, $45.9 \%$ of the patients reported diminished sexual desire, whereas 35.9\% and 36.1\% reported erectile and ejaculatory dysfunction, respectively. A total of 20 patients used risperidone, 26 olanzapine, 9 quetiapine, 13 ziprasidone, and 1 aripiprazole. More than half the sample was hyperprolactinemic, and about one fifth had prolactin levels more than 3 times the upper threshold, none of which were caused by the biologically inert macroprolactin fraction. Differences were noted among the drug groups, with risperidone-treated patients having the highest prolactin levels and the highest rate of hyperprolactinemia. Hyperprolactinemia has received new attention lately as potential long-term complications have been identified, including osteoporosis and carcinogenic effects (32). No association was found in the present study between prolactin levels and sexual dysfunction. In many case reports and few non-systematic reviews priapism was found to be associated with antipsychotic drug administration (33-38).

Particular interest should be given to the frequency of priapism. Drug-induced priapism accounts for as many as $15 \%$ to $41 \%$ of all cases, and antipsychotics are the most frequently involved drugs, followed by antidepressants and antihypertensive medications (39).

In conclusion, a therapy with antipsychotics medications have to be thoroughly considered in patients reporting sexual dysfunction. There is no definitive evidence that correlates hyperprolactinemia and sexual dysfunction, however since a high incidence of both conditions in treated psychotic patients has been reported, prolactin levels should be measured irrespective of whether SD symptoms are present or not.

\section{Anti-parkinsonian drugs}

The reported prevalence of sexual symptoms in men with Parkinson Disease (PD) ranges from 37\% to 65\% (40). Bronner et al. (41) reported that use of medications (selective serotonin reuptake inhibitors used for comorbid depression), and advanced PD stage contributed to the development of ED. It is possible that levodopa and other antiparkinsonian medications may affect sexual function in PD. However, it is not entirely clear to what extent levodopa influences sexual dysfunction in PD. Pathological hypersexuality may occur together with (42) or without delirium (43), which is attributed to the dopamine dysregulation syndrome in this disorder. Deep brain stimulation in the subthalamic nucleus has produced either improved sexual wellbeing (44) or transient mania with hypersexuality (45) in patients with PD (40). More studies are needed to better understand the etiology and probable mechanism of action of levodopa in determining sexual dysfunction in men with Parkinson Disease.

\section{Proton pump inhibitors}

A case report recently described a 42-year-old woman with previously normal sexual function who gradually developed loss of libido during treatment with esomeprazole. While taking esomeprazole, the patient's loss of libido improved with oral testosterone supplementation and deteriorated after testosterone withdrawal. Steady improvement in both sexual function and serum free testosterone concentration after discontinuation of esomeprazole was observed. Based on this evidence, proton pump inhibitors (PPI) could determine sexual dysfunction by modulation of the isoformes of cytochrome enzymes involved in testosterone metabolism (46).

Only 1 case report was found regarding sexual dysfunction induced by PPI (46). Given the large number of prescription and administrations of these medications, further investigation of the role that these drugs may play in sexual dysfunction should be conducted.

\section{PDE5 and combination therapy: \\ interaction and patient's safety}

The phosphodiesterase-5 inhibitors (PDE5i) sildenafil, vardenafil, and tadalafil are considered first-line therapy for the treatment of patients with erectile dysfunction (ED). The widespread application of PDE5i, that causes the potential for drug-drug interactions emerges as a relevant factor in determining the safety profile of PDE5i. The use of nitrates remains the only contraindidcation for all 3 PDE5i. Vardenafil is also not recommended in patients taking type 1A (such as quinidine, or procainamide) or type 3 antiarrhythmics (such as sotalol, or amiodarone) while no other major limitations have been reported for tadalafil and sildenafil. In contrast to previously reported labeling, recent studies have suggested only precaution, but not contraindication, with the concomitant use of alpha-blockers agents. In addition, precaution is also suggested in the presence of potent CYP3A inhibitors such as azole antifungals, antiretroviral protease inhibitors, or macrolide antibiotics. This is because sildenafil, vardenafil, and tadalafil are metabolized mainly via the CYP3A4 pathway. On the other hand, statins and testosterone seem to have synergic effects with PDE5i on sexual activity (47).

The safety and efficacy of the 3 currently available PDE5 inhibitors (sildenafil, tadalafil, and vardenafil) have been evaluated extensively in patients with ED and concomitant cardio vascular disease (CVD), hypertension, dyslipidemia, or diabetes with or without additional risk factors. Overall, these studies have shown similar efficacy for the 3 agents resulting in significant improvement of erectyle function in patients with any of these comorbid conditions. Their safety profile was also similar. No adverse effects on cardiac contraction, ventricular repolarization, or ischemic threshold was noted, and there was no evidence of increased cardiovascular risk from using any of these agents. However, because ED is known to be a harbinger of cardiovascular events in some men, the presence of ED should prompt investigation and intervention for cardiovascular risk factors (48).

Lastly, in a recent study of men with high systolic blood pressure who had initiated ED therapy, was showed an improvement in the systolic blood pressure control. 
After initiating therapy with PDE5i, patients were more likely to start an antihypertensive medication (17.3\%) versus stop therapy (2.3\%) and add additional antihypertensive medication to their existing therapy (42.2\%) versus decrease the number of medications (17.3\%).

Surveillance also increased with total number of systolic BP measurements increasing by $42 \%$. In conclusion, men with high systolic BP who initiated ED therapy had improvements in systolic BP control that may be related to clinically relevant behaviors, such as more aggressive monitoring and treatment with antihypertensive medications. Future research should further explore the underlying reasons and mechanisms for the observed improvements in systolic BP and whether cessation of ED therapy results in worsening BP control (49).

\section{Conclusions}

Patients affected with sexual dysfunction are often aged and assume several drugs (Figure 1).

Slabaugh et al. published a study reporting that $39.4 \%$ of patient over 65 years were exposed to multi-drug consumption during the study period (50). Elderly people are substantial consumers of medications and communitybased surveys reveal that they take an average of 2.7 to 3.9 prescription and nonprescription medications (51). Coadministration of drugs may be a factor for sexual dysfunction in single patients, and effects on sexual function may be unforeseeable. In our search we did not find any trial exploring the effects of multiple drugs prescription on sexual function. However, considering the results of available data, a negative synergic effect on sexual function is plausible. When evaluating an elderly man with $\mathrm{ED}$, medical history should be attentively focused on pharmacological therapies. The "myth" of sex-killer drugs, e.g. 5alpha reductase inhibitors or beta-blockers, that should be identified amongst a number of several "innocent" drugs possibly assumed by a single patient should be put into

\section{Figure 1.}

Median overage of drugs prescriptions by age and sex in elderly population (adapted by: EPSE Study Crhrischilles EA, et al. 1992).

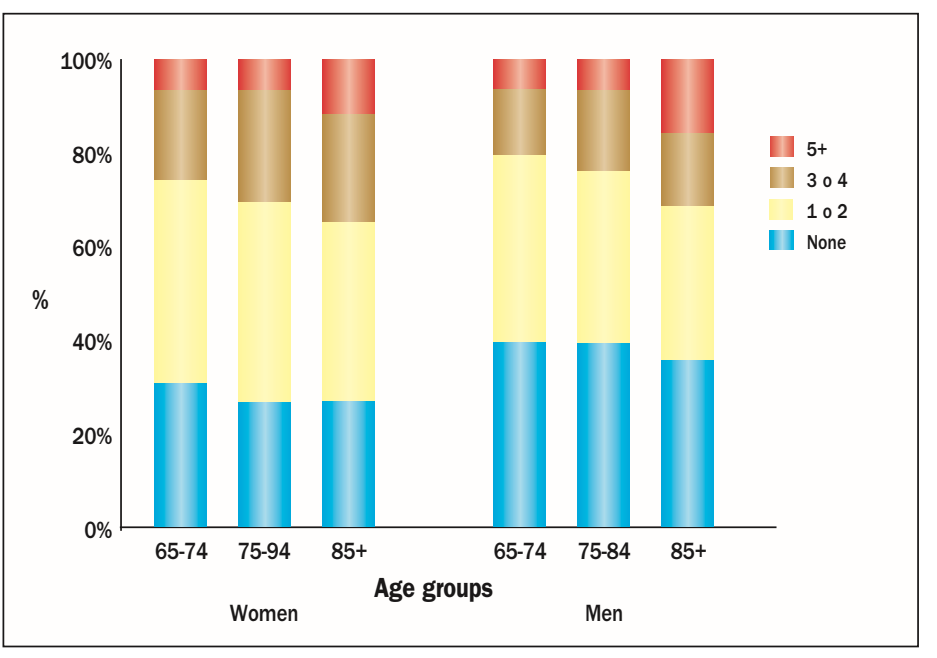

perspective. Many widely diffused drug classes that would most commonly be defined as "unsuspectable" have the potential to affect sexual function on their own, and even more so when the number of co-administered drugs makes a synergistic, multidrug impact plausible.

While Iatrogenic sexual dysfunction is prevalent in men, urological drugs are not the only drugs to be held accountable for this. Many different drugs acting on different sites and with several mechanisms of action can induce sexual dysfunction.

The drug classes involved are widely diffused and frequently assumed in combination therapies. Therefore, both general practitioners and specialists must consider the importance of pharmacological therapy in their clinical practices.

\section{REFERENCES}

1. Bancroft J. The biological basis of human sexuality. In: Human Sexuality and its problems. Edinburgh: Churchill LIvingstone. 1989; 12-127.

2. Kaplan HP. The New Sex Therapy. New York, Brunner/Mazel. 1974.

3. Shubulade S. Drugs that cause sexual dysfunction. Psychiatry. 2007; 6:111-114

4. Nagaraj AK, Pai NB, Rao S, et al. Biology of Sexual Dysfunction, Online Journal of Health and Allied Sciences. 2009; 8:1-7.

5. Mourits MJ, Bockermann I, de Vries EG, et al. Tamoxifen effects on subjective and psychosexual well-being, in a randomized breast cancer study comparing high-dose and standard-dose chemotherapy. $\mathrm{Br} \mathrm{J}$ Cancer. 2002; 86:1546-50.

6. Serretti A, Chiesa A. Treatment-emergent sexual dysfunction related to antidepressants: a meta-analysis. J Clin Psychopharmacol. 2009; 29:259-66.

7. Montejo AL, Llorca G, Izquierdo JA, Rico-Villademoros F. Incidence of sexual dysfunction associated with antidepressant agents: a prospective multicenter study of 1022 outpatients. Spanish Working Group for the Study of Psychotropic-Related Sexual Dysfunction. J Clin Psychiatry. 2001; 62(Suppl 3):10-21.

8. Bonierbale M, Lancon C, Tignol J. The ELIXIR study: evaluation of sexual dysfunction in 4557 depressed patients in France. Curr Med Res Opin. 2003; 19:114-24.

9. Reis RM, de Angelo AG, Sakamoto AC, et al. Altered sexual and reproductive functions in epileptic men taking carbamazepine. Sex Med. 2013; 10:493-9.

10. Keller J, Chen YK, Lin HC. Association between epilepsy and erectile dysfunction: Evidence from a population-based study. J Sex Med. 2012; 9:2248-55.

11. Calabro RS, Italiano D, Bramanti P, Ferlazzo E. Zonisamiderelated erectile dysfunction. J Sex Med. 2011; 8:1256-7.

12. Maschio M, Saveriano F, Dinapoli L, Jandolo B. Reversible erectile dysfunction in a patient with brain tumorrelated epilepsy in therapy with zonisamide in add-on. J Sex Med. 2011; 8:3515-7.

13. Calabrò RS, Italiano $D$, Militi $D$, Bramanti $P$. Levetiracetam-associated loss of libido and anhedonia. Epilepsy Behav. 2012; 24:283-4.

14. Lambert MV. Seizures, hormones and sexuality. Seizure. 2001; 10:319-40. 
15. Calabrò RS, Marino S, Bramanti P. Sexual and reproductive dysfunction associated with antiepileptic drug use in men with epilepsy. Expert Rev Neurother. 2011; 11:887-95.

16. Civardi C, Collini A, Gontero P, Monaco F. Vasogenic erectile dysfunction Topiramate-induced. Clin Neurol Neurosurg. 2012; 114:70-1.

17. Calabrò RS, Bramanti P, Italiano D, Ferlazzo E. Topiramateinduced erectile dysfunction. Epilepsy Behav. 2009; 14:560-1.

18. Calabrò RS. Topiramate and erectile dysfunction: Pathogenic mechanisms beyond sexual hormonal changes! Clin Neurol Neurosurg. 2012; 114:1114.

19. Ferrario CM, Levy P. Sexual dysfunction in patients with hypertension: implications for therapy. J Clin Hypertens (Greenwich). 2002; 4:424-32.

20. Fogari $R$, Preti $P$, Derosa $G$, et al. Effect of antihypertensive treatment with valsartan or atenolol on sexual activity and plasma testosterone in hypertensive men. Eur J Clin Pharmacol. 2002; 58:177-80.

21. Degroat WC, Booth AM. Physiology of male sexual function. Ann Intern Med. 1980; 92:329-331.

22. Suzuki $H$, Tominaga T, Kumagai $H$, Saruta T. Effects of first-line antihypertensive agents on sexual function and sex hormones. J Hypertens 1988; 6 (Suppl 4):S649-S651.

23. Andersen P, Seljeflot I, Herzog A, et al. Effects ofdoxazosin and atenolol on atherothrombogenic risk profile in hypertensive middleaged men. J Cardiovasc Pharmacol. 1998; 31:677-683.

24. Ko DT, Hebert PR, Coffey CS, et al. Beta-blocker therapy and symptoms of depression, fatigue, and sexual dysfunction. JAMA. 2002; $17 ; 288: 351-7$

25. Doumas M, Tsakiris A, Douma S, et al. Beneficial effects of switching from beta-blockers to nebivolol on the erectile function of hypertensive patients. Asian J Androl. 2006; 8:177-82.

26. Cocco G. Erectile dysfunction after therapy with metoprolol: the Hawthorne effect. Cardiology. 2009; 112:174-7

27. Chang SW, Fine R, Siegel D, e al. The impact of diuretic therapy on reported sexual function. Arch Intern Med. 1991; 151:2402-8.

28. Rockhold RW. Thiazide diuretics and male sexual dysfunction Drug Dev Res. 1992; 25:85-95.

29. Ekman E, Hägg S, Sundström A, Werkström V. Antihypertensive drugs and erectile dysfunction as seen in spontaneous reports, with focus on angiotensin II type 1 receptor blockers. Drug Healthc Patient Saf. 2010; 2:21-25.

30. Cutler AJ. Sexual dysfunction and antipsychotic treatment. Psychoneuroendocrinology. 2003; 28 (Suppl 1):69-82.

31. Wesby R, Bullimore E, Earle J, Heavey A. A survey of psychosexual arousability in male patients on depot neuroleptic medication. Eur Psychiatry 1996; 11:81-86.

32. Johnsen E, Kroken R, Løberg EM, et al. Sexual dysfunction and hyperprolactinemia in male psychotic inpatients: a cross-sectional study. Adv Urol. 2011; 2011:686924

33. Pais VM, Ayvazian PJ. Priapism from quetiapine overdose: first report and proposal of mechanism. Urology. 2001; 58:462.

34. Davol P, Rukstalis D. Priapism associated with routine use of quetiapine: case report and review of the literature. Urology. 2005; 66:880.

35. Andrés Prado MJ, Vidal Formoso M. J Priapism associated with quetiapine in an elderly patient Actas Esp Psiquiatr. 2006; 34:209-10.

36. Kirshner A, Davis RR. Priapism associated with the switch from oral to injectable risperidone. Clin Psychopharmacol. 2006; 26:626-8.
37. Torun F, Yilmaz E, Gümüs E. Priapism due to a single dose of quetiapine: a case report. Turk Psikiyatri Derg. 2011; 22:195-9.

38. Sinkeviciute I, Kroken RA, Johnsen E. Priapism in antipsychotic drug use: a rare but important side effect. Case Rep Psychiatry. 2012 ;2012:496364.

39. Sinkeviciute I, Kroken RA, Johnsen E. Priapism in antipsychotic drug use: a rare but important side effect. Case Rep Psychiatry. 2012; 2012:496364

40. Sakakibara R, Kishi M, Ogawa E, et al. Bladder, bowel, and sexual dysfunction in Parkinson's disease. Parkinsons Dis. 2011; 2011:924605.9

41. Bronner G, Royter V, Korczyn AD, Giladi N. Sexual dysfunction in Parkinson's disease. Journal of Sex and Marital Therapy. 2004; 30:95-105.

42. Klos KJ, Bower JH, Josephs KA, et al. Pathological hypersexuality predominantly linked to adjuvant dopamine agonist therapy in Parkinson's disease and multiple system atrophy. Parkinsonism and Related Disorders. 2005; 11:381-386.

43. Kessel BL. A case of hedonistic homeostatic dysregulation. Age and Ageing. 2006; 35:540-541.

44. Castelli L, Perozzo P, Genesia ML, et al. Sexual well being in parkinsonian patients after deep brain stimulation of the subthalamic nucleus. Journal of Neurology, Neurosurgery and Psychiatry. 2004; 75:1260-1264.

45. Romito LM, Raja M, Daniele A, et al. Transient mania with hypersexuality after surgery for high-frequency stimulation of the subthalamic nucleus in Parkinson's disease. Movement Disorders. 2002; 17:1371-1374.

46. Rosenshein B, Flockhart DA, Ho H. Induction of testosterone metabolism by esomeprazole in a CYP2C19*2 heterozygote. Am J Med Sci. 2004; 327:289-93.

47. Corona G, Razzoli E, Forti G, Maggi M. The use of phosphodiesterase 5 inhibitors with concomitant medications. Endocrinol Invest. 2008; 31:799-808.

48. Nehra A. Erectile Dysfunction and Cardiovascular Disease: Efficacy and Safety of Phosphodiesterase Type 5 Inhibitors in Men With Both Conditions. Mayo Clin Proc. 2009; 84:139-148.

49. Scranton RE, Lawler E, Botteman M, et al. Effect of treating erectile dysfunction on management of systolic hypertension. Am J Cardiol. 2007; 100:459-63.

50. Slabaugh SL, Maio V, Templin M, Abouzaid S. Prevalence and risk of polypharmacy among the elderly in an outpatient setting: a retrospective cohort study in the Emilia-Romagna region, Italy. Drugs Aging. 2010; 27:1019-28.

51. Hanlon JT, Landerman LR, Wall WE Jr, et al. Is medication use by community-dwelling elderly people influenced by cognitive function? Age Ageing. 1996; 25:190-6.

\section{Correspondence}

Ferdinando Fusco, MD

ferdinando-fusco@libero.it

Marco Franco, MD (Corresponding Author)

marcofranco87@gmail.com

Nicola Longo, MD

Alessandro Palmieri, MD

Vincenzo Mirone, MD

Department of Neurosciences, Reproductive Science and Odontostomatology - Federico II University, Naples, Italy 